\title{
A Complementary Method for Production of Tetraploid Crassostrea gigas Using Crosses Between Diploids and Tetraploids with Cytochalasin B Treatments
}

\author{
Helen McCombie ${ }^{1 *}$, Christophe Ledu ${ }^{1,2}$, Pascal Phelipot $^{1}$, Sylvie Lapègue ${ }^{1}$, Pierre Boudry ${ }^{1}$ and \\ André Gérard ${ }^{1,2}$
}

1 Institut Français pour la Recherche et Exploitation de la Mer (IFREMER), Laboratoire d' Génétique et

Pathologie, La Tremblade, 17390, France

2 Present address: IFREMER, Centre de Nantes, Nantes Cedex 03, 44311, France

*: Helen McCombie Email: hmccombi@ifremer.fr Phone: (+ 33) 05-46-76-26-10 Fax: (+ 33) 05-46-76-26-11

\begin{abstract}
We present a new method to produce tetraploid Crassostrea gigas by cytochalasin B inhibition of polar body 2 expulsion in diploid females crossed with tetraploid males. This offers a means of direct introgression of genetic characters from selected diploid to tetraploid lines, avoiding a triploid step. Offspring larval ploidy shifted over time and depended on size, with tetraploids more frequent among the smaller larvae and triploids among the large. Viable tetraploids were found at 4 and 6 months, indicating the technique was successful. The possibility that gynogenesis occurred was tested by microsatellite analysis to confirm the presence of paternally inherited alleles. These were present in all animals of the $2 n \times 4 n+C B$ (female first) cross. However, a $4 n \times 2 n+C B$ cross produced triploids, including some gynogens. Our method illustrates for the first time that diploid $\mathrm{C}$. gigas eggs, if selected for large size, can give viable tetraploid offspring.
\end{abstract}

Keywords Crassostrea gigas - polyploidy - cytochalasin B - polar body - gynogenesis - oyster breeding 


\title{
A Complementary Method for Production of Tetraploid Crassostrea gigas Using Crosses Between Diploids and Tetraploids with Cytochalasin B Treatments
}

\author{
Helen McCombie, ${ }^{1}$ Christophe Ledu, ${ }^{1}$ Pascal Phelipot, ${ }^{1}$ Sylvie Lapègue, ${ }^{1}$ Pierre Boudry, ${ }^{1}$ \\ André Gérard ${ }^{1,2}$
}

${ }^{1}$ Institut Français pour la Recherche et Exploitation de la Mer (IFREMER), Laboratoire d' Génétique et Pathologie, 17390 La Tremblade, France

${ }^{2}$ IFREMER, Centre de Nantes, 44311 Nantes Cedex 03, France

Received: 8 April 2004 / Accepted: 16 September 2004 / Online publication: 5 May 2005

\begin{abstract}
We present a new method to produce tetraploid Crassostrea gigas by cytochalasin B inhibition of polar body 2 expulsion in diploid females crossed with tetraploid males. This offers a means of direct introgression of genetic characters from selected diploid to tetraploid lines, avoiding a triploid step. Offspring larval ploidy shifted over time and depended on size, with tetraploids more frequent among the smaller larvae and triploids among the large. Viable tetraploids were found at 4 and 6 months, indicating the technique was successful. The possibility that gynogenesis occurred was tested by microsatellite analysis to confirm the presence of paternally inherited alleles. These were present in all animals of the $2 n \times 4 n+C B$ (female first) cross. However, a $4 n \times 2 n+C B$ cross produced triploids, including some gynogens. Our method illustrates for the first time that diploid C. gigas eggs, if selected for large size, can give viable tetraploid offspring.
\end{abstract}

Key words: Crassostrea gigas - polyploidy cytochalasin B - polar body - gynogenesis oyster breeding

\section{Introduction}

Polyploid oysters have become increasingly important for aquaculture of Crassostrea gigas following findings that triploids (3n) offered quicker growth

Correspondence to: Helen McCombie; E-mail: hmccombi@ ifremer.fr over their natural diploid (2n) counterparts and had high levels of infertility (reviewed by Nell, 2002). Triploids bred in hatcheries come most commonly from crosses of diploid females with artificial tetraploid males (Guo et al., 1996). Although triploids were originally bred with direct chemical induction on diploid $\times$ diploid crosses (e.g., Downing and Allen, 1987; Yamamoto et al.,1988; Gérard et al., 1999), this method is less efficient and involves the use of toxic chemicals on animals for the market. The production and maintenance of tetraploid $(4 n)$ oysters has therefore become vital to the supply of triploids.

Successful tetraploid induction has been achieved in $C$. gigas by chemical treatment on a cross of a fertile triploid female with a diploid male (Guo and Allen, 1994; Figure 1). However, fertile 3n animals can be difficult to find as sterility is a characteristic of this ploidy class and eggs present in such individuals may be of limited number and viability (Guo and Allen, 1994b; Que et al., 1997). Progenies produced by this technique yield a highly variable proportion of tetraploids (e.g., 7\%-96\%, Eudeline et al 2000). Once tetraploids are produced, though, their fertility means that they may be interbred, $4 n \times 4 n$, to produce $4 n$ progenies. However, non-4n animals have been reported in such progenies, which could be due to unbalanced chromosome numbers in the female gametes or to somatic chromosome loss (McCombie et al., in press).

Many studies have shown heritable variation for traits of interest in oyster aquaculture (e.g., Ernande et al., 2004). Since the development of hatcherybased propagation of oysters (Robert and Gérard, 1999), selective breeding programs have been initiated in several countries (for review, see Sheridan, 1997; Nell et al., 2000; Ward et al., 2000; Langdon 

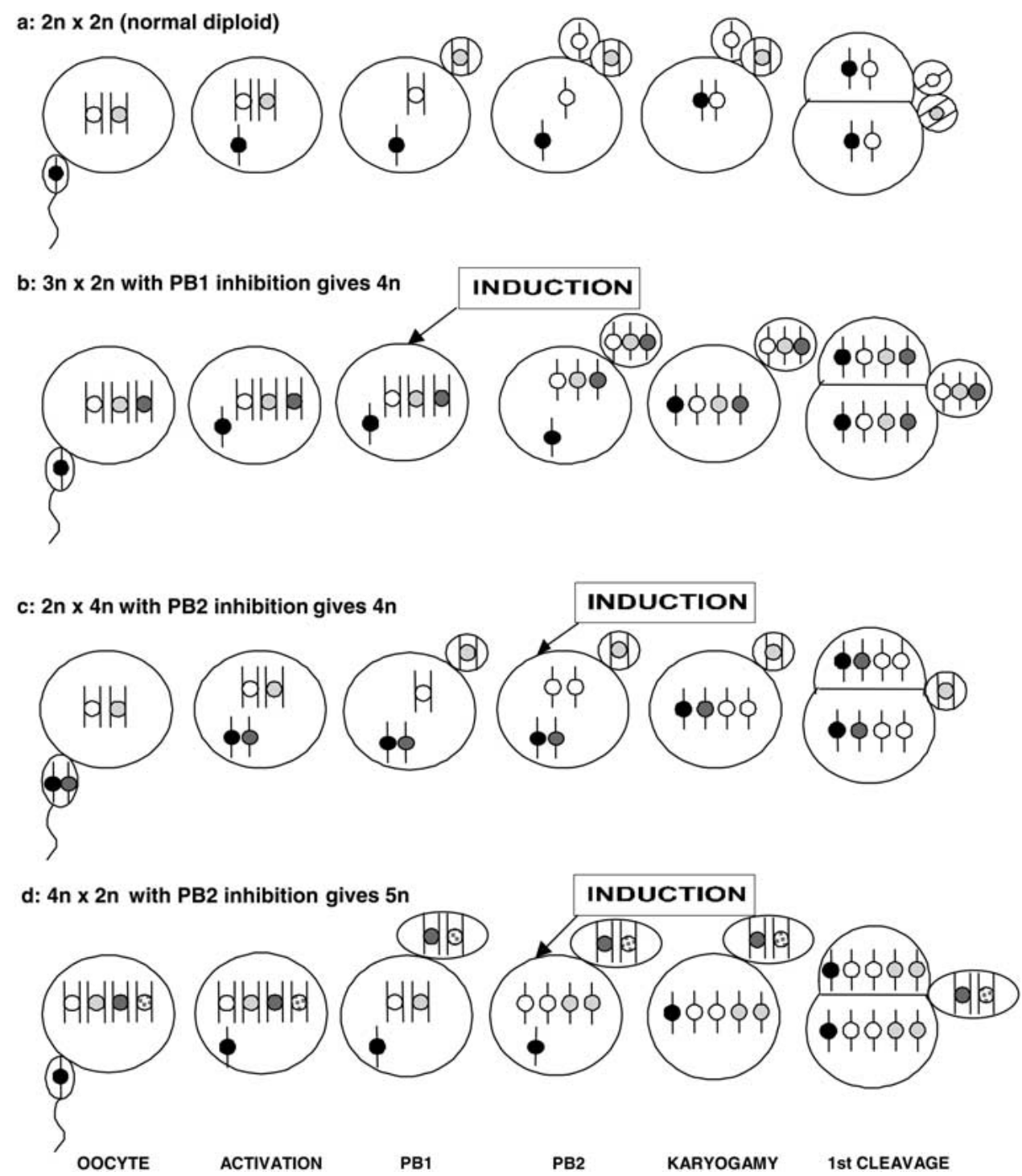

Fig. 1. Diagram of embryogenic events in a natural cross of diploid-diploid $(2 \mathrm{n} \times 2 \mathrm{n})$ Crassostrea gigas (a) and in crosses with ploidy change induced by cytochalasin B treatment at different stages: tetraploid (4n) induction from triploid (3n) females $\times$ diploid males $(\mathbf{b})$, hypothetical schema of tetraploid production from diploid females $\times$ tetraploid males crosses performed in this study (c), hypothetical schema of pentaploid (5n) production from tetraploid females $\times$ diploid male crosses performed in this study (d). The female parent is mentioned first in all the cross names.

et al., 2003). Thus far, these programs have used only diploid stocks. Indeed, selective breeding of tetraploids is known to be complex and slow compared with that of diploids (Kearsey and Pooni, 1996). It is therefore unlikely that selective breeding of tetraploid oysters will efficiently contribute to the development of oyster production in the near future. Furthermore, selection on tetraploids is also restricted by the strict quarantine conditions observed for their culture in some countries (Boudry and Chatain, 1999).

Nevertheless, the selective breeding of $4 \mathrm{n}$ lines and improvement of their genetic characteristics are important steps toward the production of genetically improved triploids. It is therefore necessary to have a viable means of generating tetraploids de novo from improved 2n stocks or, alternatively, of integrating genetic material from $2 \mathrm{n}$ animals into existing $4 \mathrm{n}$ families. The use of eggs from $3 \mathrm{n}$ females is dependent on the fertility of these maternal progenitors and the quality of their gametes. In aquaculture, fertility is not a desirable characteristic of $3 \mathrm{n}$ animals, and the deliberate selection of fertile $3 n$ to supply parents for $4 \mathrm{n}$ stocks may present a problem of fertility in subsequent $3 \mathrm{n}$ progeny of the $4 \mathrm{n}$ thus produced. Indeed there are already indications that the characteristic of high fertility can be passed from triploid to tetraploid (Guo and Allen, 1997). 
Table 1. Mating Design for Induction Trials and Control Famlies Using Pools of Gametes from the Same Parents $(n=\text { number of individuals in the parental pools })^{\text {a }}$

\begin{tabular}{lll}
\hline & Diploid females $(n=2)$ & Tetraploid females $(n=3)$ \\
\hline Diploid males $(n=2)$ & Batch 1: $2 \mathrm{n} \times 2 \mathrm{n}$ Control & Batch 4: $4 \mathrm{n} \times 2 \mathrm{n}+\mathrm{CB}$ \\
Tetraploid males $(n=3)$ & Batch 3: $2 \mathrm{n} \times 4 \mathrm{n}+\mathrm{CB}$ & Batch $2: 4 \mathrm{n} \times 4 \mathrm{n}$ Control \\
\hline
\end{tabular}

${ }^{a}$ The female parent is mentioned first in all the names. Batches 1 and 2 represent the controls, which were diploid ( $\left.2 \mathrm{n}\right)$ and tetraploid ( $\left.4 \mathrm{n}\right)$, respectively. Batch 3 tests the new induction cross, which hypothetically produces tetraploids from $2 \mathrm{n}$ females $\times 4 \mathrm{n}$ males with cytochalasin $(\mathrm{CB})$ treatment. Batch 4 hypothetically produces pentaploids $(5 \mathrm{n})$ from $4 \mathrm{n}$ females $\times 2 \mathrm{n}$ males with CB treatment.

An alternative means of generating tetraploids in bivalve mollusks is directly from diploids by inhibiting the expulsion of both polar bodies (Scarpa et al., 1993). The use of diploids would allow animals grown and selected under normal culture conditions to be converted directly into tetraploids. However, direct induction from diploids has generally resulted in low percentages of tetraploids produced per cross and low survival rates (Scarpa et al., 1993; Peruzzi and Guo, 2002). Two reasons for this might be (1) the longer chemical treatment, which could increase toxic effects, and (2) the hypothesis proposed by Guo and Allen (1994a), that limited diploid egg size does not allow correct early divisions with a tetraploid genome, which is too large, and the results are lethal for subsequent development.

Another means of exploiting genetic characters from diploids in tetraploid lines would be the introgression of this material into $4 \mathrm{n}$ families. In this report we present a new method for achieving this objective. We crossed $2 \mathrm{n}$ females with $4 \mathrm{n}$ males: $2 \mathrm{n} \times$ $4 \mathrm{n}$ (the female is mentioned first in all cross names). Such a cross would normally produce triploid offspring and is the basis of crosses made in hatcheries for triploid production. In our case, however, we applied a cytochalasin B (CB) treatment to the fertilized eggs (activated oocytes) to inhibit the expulsion of the second polar body with the objective of adding an extra haploid genome and producing tetraploids (Figure 1). A similar principle has been used in rainbow trout (Chourrout et al., 1986), although heat shocks rather than $\mathrm{CB}$ were used to retain the second polar body. Retention of the second polar body, rather than the first, is less likely to engender complications in subsequent development (Gérard et al., 1999), and in the present context, allows a balanced genetic contribution from both maternal and paternal parents (Pandian and Koteeswaran, 1998). To counter potential negative effects of small diploid egg size, we exploited natural variation in egg size between diploid females to find females with large eggs to serve as maternal parents in the induction cross. Finally, to further understand the mechanism of this induction method, we investigated the possibility of breeding pentaploids by making the same
CB treatment on a cross between tetraploid females and diploid males (Figure 1).

\section{Materials and Methods}

Selection of Progenitors and Mating Design. Diploid and tetraploid Crassostrea gigas to serve as progenitors were brought to sexual maturation in the IFREMER hatchery at La Tremblade. The ploidy of the tetraploid parents was confirmed using image analysis on Feulgen-stained nuclei obtained from gill tissue (Gérard et al., 1994). The diploid females selected were those with the largest egg size after the eggs were stripped on the day the cross was made. Mean egg diameter was measured using a Nikon profile projector on a sample of 10 eggs per animal on 6 diploid females. Larger sample sizes could not be taken because of time constraints on the viability of eggs after stripping. Mean egg size per female varied between 53 and $60 \mu \mathrm{m}$. The 2 females with the largest eggs (mean, $60 \mu \mathrm{m})$ were selected to be the female diploid progenitors.

The crossing design, shown in Table 1, included diploid and tetraploid control progenies in addition to the treatment batches. Pools of stripped gametes were made for each ploidy and sex group. Two animals were used to give each diploid group, while 3 were necessary for the tetraploids because of generally lower gamete numbers in tetraploids. Samples of gill tissue from the chosen parents were preserved in $100 \%$ ethanol for use in future DNA analyses. The 4 batches each had 15 million oocytes with spermatozoa (oocyte $=$ spermatozoon ratio $=1: 200$ ) in $1 \mathrm{~L}$ seawater at $25^{\circ} \mathrm{C}$, maintained at this temperature in a water bath.

Cytochalasin B Treatments and Samples for Embryogenesis Study. All treatments and samplings were made at precise points in time after fertilization (fertilization $=0$ minute). The 2 batches with CB were treated at 20 minutes for a duration of 20 minutes to inhibit the expulsion of the second polar body. CB (Sigma C-6762), dissolved in (dimethylsulfoxide DMSO, Lab-Osi D-770) was added to each of the treatment beakers to produce final con- 
centrations of $1 \mathrm{mg} / \mathrm{L}$ After the treatment period, the $\mathrm{CB}$ was removed by filtration on a $10 \mu \mathrm{m}$ gauze, and the embryos were rinsed in DMSO $(1 \mathrm{ml} / \mathrm{L})$ in seawater for 15 minutes. They were subsequently refiltered and put in clean filtered seawater.

Samples for embryo study were taken at fertilization and every 5 minutes thereafter until 60 minutes, then every 10 minutes until 80 minutes after fertilization. At each interval, $1 \mathrm{ml}$ was taken and immediately fixed in formaldehyde in a glucosamine-acetate (GA) buffer (Dube et al., 1985). These samples were later stained with the fluorochrome Hoeschst 33258 (Sigma B2883) and examined by epifluorescence with an Olympus BH-2 UV microscope. One hundred embryos were studied per sample, and these were classified according to their expulsion of polar bodies and division status.

Larval Rearing, Nursing, and Growth. The embryos were transferred to $300-\mathrm{L}$ volume fiberglass tanks of filtered seawater 90 minutes after fertilization. These tanks served as a growing environment until fixation. The water was oxygenated by a continuous bubble-through air supply, and the larvae were fed daily, starting at 24 hours after fertilization, with a cultured supplement of phytoplankton Isochrysis galbana, Chaetoceros calcitrans (formerly pumillum), and Tetraselmis suecica. At 24 hours the larvae were collected by sieving on a $45-\mu \mathrm{m}$ nylon mesh, and the number of larvae that had reached D-stage was estimated for each batch. The water in the tanks remained at a mean of $22.5^{\circ} \mathrm{C}$ (minimum, $22.1^{\circ} \mathrm{C}$, maximum $23.5^{\circ} \mathrm{C}$ ) and $32.9 \%$ salinity (minimum $29.4 \%$, maximum, $34.1 \%$ ) and was changed every 2 days by catching the larvae on a nylon mesh, the gauge of which was adjusted with the growth of the larvae. The number of live larvae in each batch was estimated at each water change (method described in Ernande et al., 2003). This allowed us to detect any abnormal mortality or developmental pattern that could have occurred. Although there was no replication of tanks within batches at the larval rearing stage because of technical constraints in the hatchery, a previous study conducted in the same rearing room had shown that intertank effects were negligible (Ernande et al., 2003).

The larvae were put to settle when they had attained the pediveliger stage (16-22 days). They were transferred into $150-\mu \mathrm{m}$ mesh-bottomed trays with ground oyster shell as a medium for fixation. These trays were kept in raceways filled with recirculating seawater containing phytoplankton supplements. The oysters remained in a quarantine area of the hatchery, and trays were replaced with others with larger-gauge mesh as the animals grew. Water in the raceways was changed 4 times a day automatically, and all the water running out after renewal was treated with ozone to prevent any escape of polyploids or their gametes into the natural environment.

Ploidy Level Measurement. Samples of larvae were taken from each batch at 24 and 72 hours after fertilization. Preparations for ploidy analysis were made following a modified protocol of Gérard et al. (1994). Each sample was concentrated with a drop of $37 \%$ formol, ground in a Dounce tube, and left 15 minutes in a hypotonic shock of 50:50 seawaterdistilled water. Fixation was made in 4 changes of Carnoy's fixative (1:3 glacial acetic acid-absolute ethanol) at $4^{\circ} \mathrm{C}$ for 10,10 , and 15 minutes before storage at $4^{\circ} \mathrm{C}$. Larvae were retrieved between changes by centrifugation at $1000 \mathrm{rpm}$. Drops of ground fixed larvae were placed in 50\% acetic acid and vortexed for disassociation. These were dropped onto microscope slides at $44^{\circ} \mathrm{C}$, and the excess liquid was removed with a Pasteur pipette. The dry slides were Feulgen-stained and studied with an image analysis system (Samba) of a microscope linked to a computer with a program to calculate ploidy levels based on optic densities.

Ploidy measurements were also made on young oysters at 4 months and 6 months. The first of these measurements was a test of ploidy alone, to verify the success of the experiment and survival of the tetraploids, for which 10 animals were sampled from each batch. At 6 months, a larger ploidy test of $n=24$ animals per batch was accompanied by microsatellite analysis of parentage of the 2 CB-treated progenies to ascertain whether animals had indeed been sexually produced or were gynogens (parthenogenic individuals that develop following activation by a male but which contain only maternal genetic material). Both the 4-month and 6-month ploidy tests were destructive, and image analysis was performed on Feulgen-stained nuclei made from gill tissue printing on microscope slides as in Gérard et al. (1994).

Microsatellite Analyses. Parentage analyses were performed on 24 individuals from each of the CB-treated batches (batch 3:2n $\times 4 n$ and batch $4: 4 n \times$ 2n) using highly variable microsatellite markers. The objective was to determine whether or not the paternal parents had participated in the cross, first because our initial ploidy results had revealed diploids in batch 3 , and it is known that diploid gynogens may be produced by $\mathrm{CB}$ treatments in this species (Guo et al., 1993). Second, results from our embryogenesis study had also indicated some double 


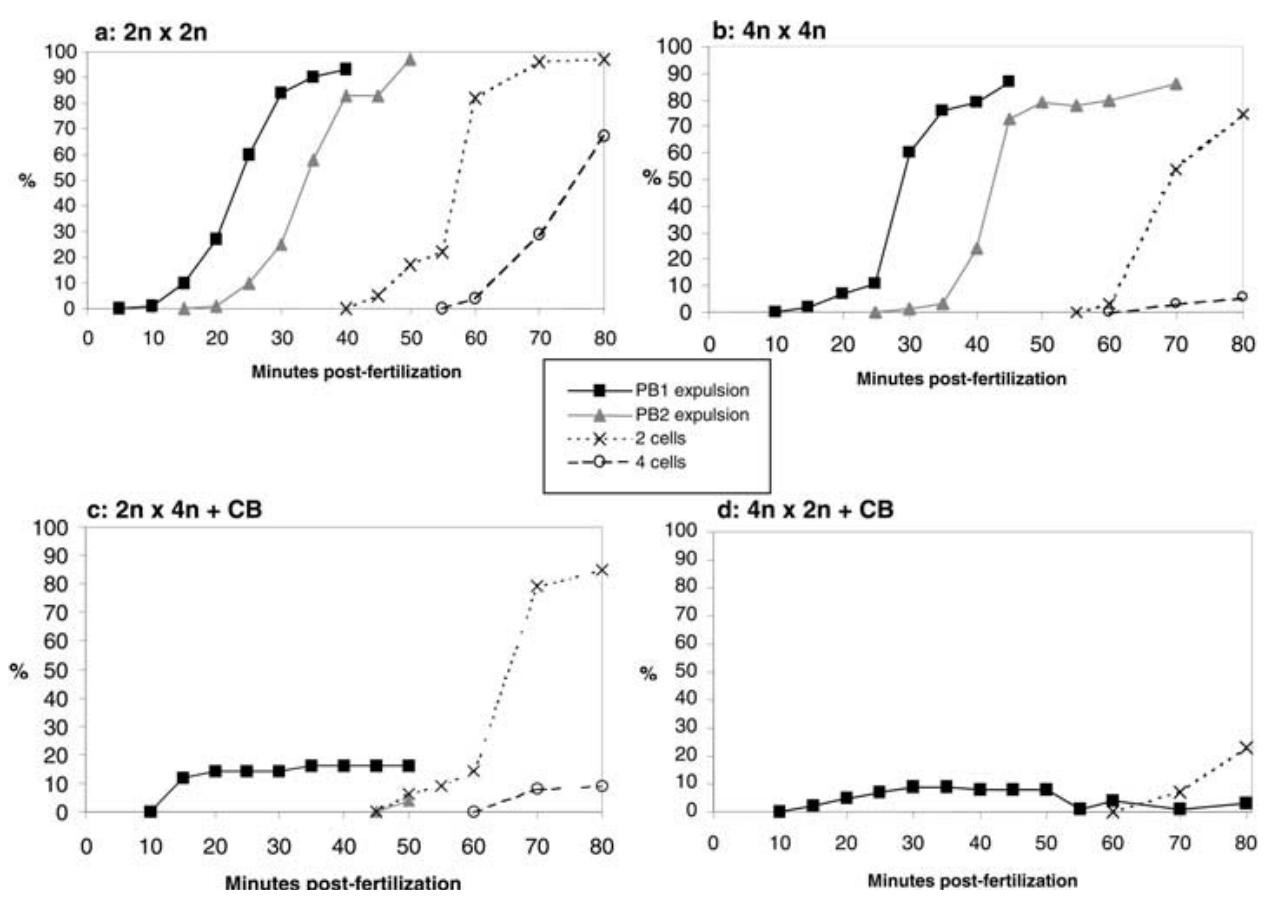

Fig. 2. Embryogenic development of crosses performed in this study from $0-80$ minutes after fertilization. Diploid $(2 \mathrm{n}) \times$ diploid control $(\mathbf{a})$, tetraploid $(4 \mathrm{n}) \times$ tetraploid Control $(\mathbf{b})$, diploid $\times$ tetraploid with cytochalasin B (CB) treatment at 20-40 minutes $(\mathbf{c})$, tetraploid $\times$ diploid with cytochalasin B treatment at 20-40 minutes (d). PB1 is first polar body; PB2, second polar body; $n=100$.

polar body retention, which could lead to tetraploid gynogens (Guo et al., 1993; Ledu and McCombie, 2003).

Parentage analysis was performed by hand. For batch 3:2n $\times 4 \mathrm{n}+\mathrm{CB}, 2$ markers, CG49 (Magoulas et al., 1998) and $L 10$ (Huvet et al., 2000), were necessary to distinguish the maternal and paternal parents and therefore to identify contribution of a father. In batch $4: 4 n \times 2$ n, only CG49 was necessary.

Total DNA was extracted from gill tissue of the animals using a phenol-chloroform extraction. Amplification with infrared detection-labeled microsatellite primers was used to obtain multiple copies of the alleles present in each individual. The amplification reactions were made with $5.0 \mu \mathrm{l}$ diluted DNA (20-40 ng), $1.0 \mu \mathrm{l} 10 \times$ PCR buffer, $0.5 \mu \mathrm{l}$ (1 pmol) labeled forward primer, $0.5 \mu \mathrm{l}(1 \mathrm{pmol})$ reverse primer, $0.2 \mu \mathrm{l}$ Silver Star DNA polymerase, 0.5 $\mu 12 \mathrm{mM}$ dNTPs, $1.3 \mu \mathrm{l} \mathrm{H}_{2} \mathrm{O}$. The heat treatments were as follows: 5 minutes of denaturation at $95^{\circ} \mathrm{C}$, followed by 30 cycles of: 20 seconds at $95^{\circ} \mathrm{C}, 20$ seconds annealing at $53^{\circ}$ or $55^{\circ} \mathrm{C}$ for CG49 or L10, respectively, and 30 seconds at $72^{\circ} \mathrm{C}$. A final step consisted of 3 minutes at $72^{\circ} \mathrm{C}$. PCR products were denatured once more at $95^{\circ} \mathrm{C}$ with stop buffer before introduction into a wafer-thin acrylamide gel on a Li-Cor IR 24200 DNA sequencer. Microsatellite band patterns were revealed by $800 \mathrm{~nm}$ laser excitation of the marker on the DNA fragments and stored as an image file on a computer connected to the sequencer. Banding patterns were analyzed using the Gene Profiler software package.

\section{Results}

Embryo Development. The scoring of successive stages of embryogenesis in the controls and treated samples showed a typical series of events for the diploid control (Figure 2, a) whereby expulsion of the first polar body (PB1) started between 5 and 10 minutes after fertilization, followed by the second polar body (PB2) about 10 minutes later. The first mitotic divisions followed within the first hour of development. The percentage of cells at each stage evolved over time, and variation in stages attained in the crosses can be seen in Figure 2.

The $4 \mathrm{n} \times 4 \mathrm{n}$ cross showed similar development to the diploid control although events were slower (Figure 2, b) compared with the diploid, a result that follows observations made by Guo and Allen (1997).

In the treated crosses (Figures 2, c and d), very few PB2 were observed, as was expected with the treatment made to inhibit this expulsion. There were also fewer PB1 than would be expected for treatments intended to inhibit only PB2. Owing to slower development than expected and variation in stage at a single point in time, we may have inhibited both PB1 and PB2 in a number of embryos. A 
Table 2. Number of Developing Larvae at 24 and 72 Hours After Fertilization with Percentage Survival Relative to Number of Eggs (primary oocytes) at Fertilization ${ }^{\text {a }}$

\begin{tabular}{lllll}
\hline & \multicolumn{2}{c}{ No $C B$} & & \multicolumn{1}{c}{ CB treatment } \\
\cline { 2 - 3 } & $\begin{array}{l}\text { Batch 1: } \\
2 n \times 2 n \text { Control }\end{array}$ & $\begin{array}{l}\text { Batch 2: } \\
4 n \times 4 n \text { Control }\end{array}$ & & $\begin{array}{l}\text { Batch 3: } \\
2 n \times 4 n+C B\end{array}$ \\
\hline Nb.eggs & $15,000,000$ & $15,000,000$ & $15,000,000$ & $15,000,000$ \\
Nb. D-larvae 24 h & $9,500,000^{\mathrm{b}}$ & $3,000,000$ & 597,000 & $4,000,000$ \\
\% Survival 24 h & 63.00 & 20.00 & 3.98 & 26.66 \\
Nb. Larvae 72 h & $1,069,500$ & 763,000 & 164,800 & 481,300 \\
$\%$ Survival 72 h & 45.16 & 5.09 & 1.10 & 3.1 \\
\hline
\end{tabular}

${ }^{a}$ Batches 1 and 2 represent the controls, which were diploid $(2 n)$ and tetraploid $(4 n)$, respectively. Batch 3 tests the new induction cross, which hypothetically produces tetraploids from $2 \mathrm{n}$ females $\times 4 \mathrm{n}$ males with cytochalasin $\mathrm{B}$ (CB) treatment. Batch 4 hypothetically produces pentaploids $(5 \mathrm{n})$ from $4 \mathrm{n}$ females $\times 2 \mathrm{n}$ males with $\mathrm{CB}$ treatment.

${ }^{\mathrm{b}}$ This number was reduced to $1,500,000$ at $24 \mathrm{~h}$ to reduce competition, $\%$ survival at $72 \mathrm{~h}$ is adjusted accordingly.

subsequent normal first division was observed in both these batches, although this was slightly later, which may be a secondary effect of CB (Scarpa et al., 1993).

Larval Development and Ploidy. At 24 and 72 hours, the number of live D-stage larvae differed between the batches. Survival was variable between batches, and by 72 hours the treated batches showed lower percentage survival than their respective counterpart control crosses made with the same female pools (Table 2). This have been due to toxic effects of $\mathrm{CB}$ or poor survival of animals of certain ploidy classes.

The ploidy of larvae at 24 hours was at the expected levels, with a peak at $2 \mathrm{n}$ for the progeny of the $2 n \times 2 n$ cross, which was used as a control for the other samples, and one at $4 n$ for the $4 n \times 4 n$ cross (Figure 3 , a). The $2 \mathrm{n} \times 4 \mathrm{n}$ cross with CB treatment produced a wide peak with a principal point at $4 \mathrm{n}$ (Figure $3, b$ ), while the $4 n \times 2 n$ cross with CB treatment produced peaks at $2 n, 4 n, 5 n, 7 n$, and $9 n$. The result of the $2 n \times 4 n+C B$ cross implies that at this stage the treatment had had the intended result: to produce tetraploid offspring. The variety of ploidy results produced in the other treated cross, $4 n \times 2 n+$ $\mathrm{CB}$, could have arisen from treatment affecting a series of different embryo stages, accidental double polar body retention, and the increased capacity of the tetraploid eggs to hold multiple haploid genomes. At 72 hours the largest and the smallest portions of the batches by size of larvae were separated on $45 \mu \mathrm{m}$ and $70 \mu \mathrm{m}$ nylon mesh, respectively. In the $4 n \times 4 n$ cross, both the small and large-sized larvae had peaks at $4 \mathrm{n}$ (Figures 4 , a and b). In the $2 \mathrm{n} \times$ $4 n+C B$ batch, it was the small larvae that gave a principal peak at $4 \mathrm{n}$ (Figure 4, c), while the larger larvae were predominately $3 \mathrm{n}$ with a smaller peak at $4 \mathrm{n}$ (d). This may imply simple size differences between the ploidy levels or an underlying difference in vigor at this stage. In the $4 n \times 2 n+C B$ batch, the ploidy levels superior to $5 \mathrm{n}$ had disappeared at 72 hours, and the predominant peaks of both size classes were at $5 \mathrm{n}$ (Figures 4 , e and $\mathrm{f}$ ).

Ploidy of Juveniles. A test of ploidy was made at 4 months after the crosses, on juvenile animals. Ten animals only were taken per batch because tests at this age are destructive. The results, showing expected values for the untreated crosses and a mixture of animals of different ploidy $(2 n, 3 n$, and $4 n)$ for the $2 n \times 4 n+C B$, are shown in Table 3 . For the $4 n \times 2 n+$ $\mathrm{CB}$ batch, the values obtained implied that all the animals were triploid or had a ploidy between triploid and tetraploid. Another 10 animals were screened and produced similar results, except for one that showed evidence of mosaicism (Table 3). There were no $5 \mathrm{n}$ in this sample despite predominance of this ploidy class at the early larval stages.

At 6 months, a ploidy test of the treated batches showed similar results, with classes $2 n, 3 n$, and $4 n$ present in the $2 n \times 4 n+C B$ batch and $3 n$ only in the $4 n \times 5 n+$ CB batch (Table 3). There was only a slight increase in the proportion of $3 n$ in the $2 n \times 4 n+C B$ batch relative to results at 4 months.

Microsatellite Identification of Gynogens and Sexually Produced Juveniles. Within the $2 n \times 4 n+$ CB treated cross, although there were 3 different ploidy levels $(2 n, 3 n$, and $4 n)$ shown by the image analysis, all animals subsequently screened using microsatellites showed evidence of a paternal contribution, including the diploids. The $2 \mathrm{n}$ ploidy itself is surprising considering that a cross between $2 \mathrm{n}$ and $4 n$ parents normally gives 3 n (Figure 5, a), a ploidy level superior to the diploid level, even before the results of polar body inhibition are considered. The presence of the paternal alleles proves that these diploid individuals were not gynogens, which could otherwise have provided an explanation for the low 

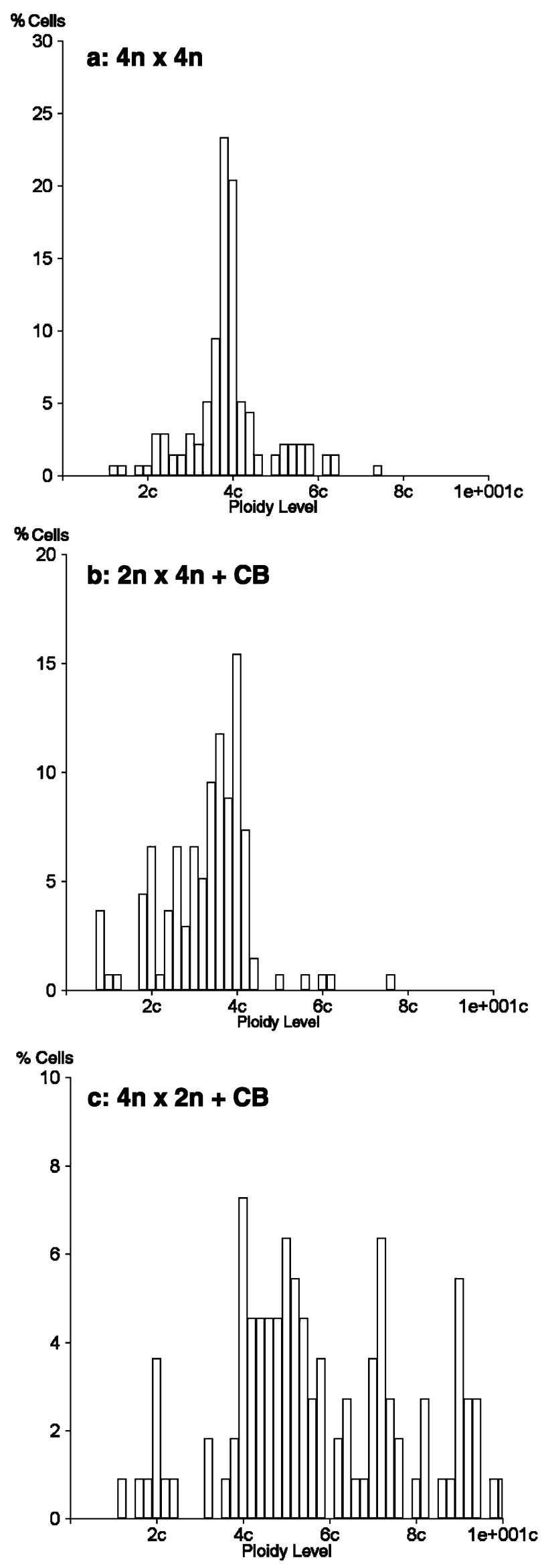

Fig. 3. Ploidy levels of larvae at 24 hours based on image analysis of Feulgen-stained nuclei from pools of ground larvae compared with a diploid control. Tetraploid $\times$ tetraploid control $(\mathbf{a})$, diploid tetraploid with cytochalasin B treatment $(\mathbf{b})$, tetraploid $\times$ diploid with cytochalasin B treatment $(\mathbf{c}) ; n=100$ to 150 .

ploidy level. The presence of the paternal alleles in the tetraploids shows that these were not gynogens either and that they were most probably induced by the suppression of the second polar body as intended in this experiment.

Gynogens were, however, found in the other treated cross of $4 n \times 2 n+C B$. 6 animals out of 24 , tested at 6 months, were found to have no paternal contribution as judged by the microsatellite alleles of the diploid fathers. These gynogenetic animals had $3 n$ ploidy profiles, as did all other animals tested in this batch at 6 months. The presence of maternal alleles in all animals tested in the $4 n \times 2 n+C B$ cross means there was no evidence for androgenesis (embryogenic development with the paternal nuclear genetic material only in the fertilized egg, from which the female nuclear genetic material has been rejected) in this batch. However, one of the diploid animals in the $2 n \times 4 n+C B$ cross had only paternal alleles of both markers, implying that the diploid sperm (from the tetraploid father) had achieved androgenesis in this case.

\section{Discussion}

Different Ploidy Classes Produced by CB Treatments. Our experiment revealed that different crosses exposed to CB treatments for PB2 suppression produced first larvae and later juveniles of different ploidy levels. Mixtures of ploidy classes were found in both treated batches at different times. Such mixed ploidy results have been found in many previous studies on ploidy manipulation in Crassostrea sp. (e.g., Stanley et al., 1981; Yamamoto et al., 1988; Guo and Allen, 1994a; Gérard et al., 1999). One reason they may be produced from the same treatment on the same cross is differential development between the embryos at the moment of treatment, leading to different effects on the development of these individuals. At the moment that the $\mathrm{CB}$ treatment was applied in batch $2 n \times 4 n+C B$, according to embryogenesis data (Figure 2, c), the following stages were present: unfertilized eggs, meiosis I completed (PB1 expulsed). This means that the treatment could have prevented expulsion of PB2 in some animals as intended, but that PB1 expulsion could have been inhibited in others. The PB1 

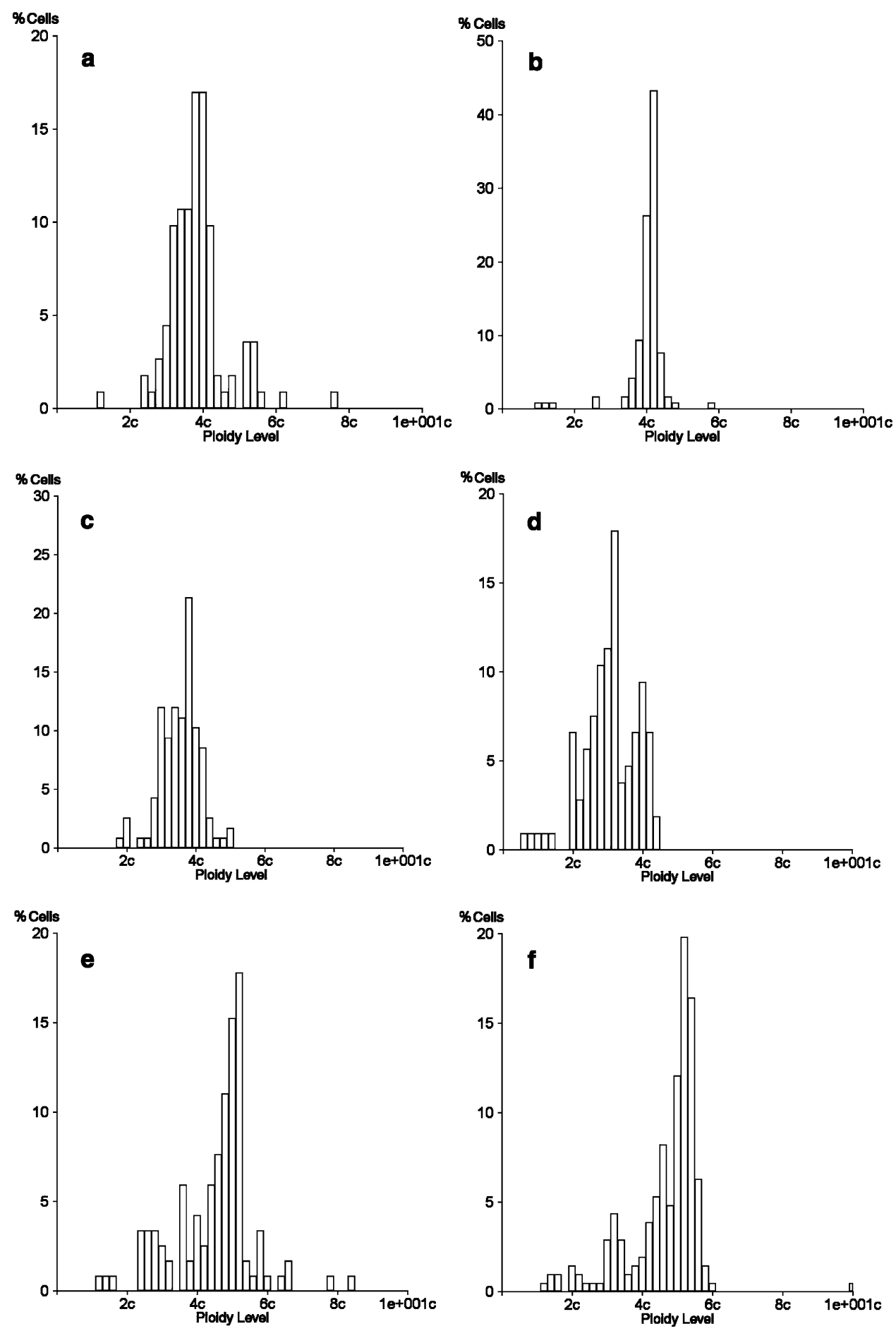

Fig. 4. Ploidy levels of larvae at 72 hours based on image analysis of Feulgen-stained nuclei from pools of ground larvae compared with a diploid control. Larvae were divided by size by sieving on a $45-\mu \mathrm{m}$ screen for the small group $(\mathbf{a}, \mathbf{c}, \mathbf{e})$ and a $70-\mu \mathrm{m}$ screen for the large group $(\mathbf{b}, \mathbf{d}, \mathbf{f})$.

Tetraploid $\times$ tetraploid $(\mathbf{a}, \mathbf{b})$, diploid $\times$ tetraploid with cytochalasin B treatment $(\mathbf{c}, \mathbf{d})$, tetraploid $\times$ diploid with cytochalasin B treatment $(\mathbf{e}, \mathbf{f})$; $n=100-150$.

expulsion pattern in Figure 2, c also suggests that the first polar body may have been inhibited in part of this batch. In some cases both PBs could have been retained because of the proximity of these two events (Figure 2,a). These possible developmental scenarios are illustrated in Figure 5. Variation in stage of development within a batch of eggs from a single female oyster has already been reported
(Longwell and Stiles, 1968), and such effects were probably enhanced by using a pool of females rather than a single individual as suggested by Eudeline et al. (2000). The retention of PB1 can produce diverse ploidy results because a mixture of chromosome segregation patterns occur (Guo et al., 1992a, 1992b), which could also contribute to the results we observed. 
Table 3. Ploidy Results of the Four Batches at 4 Months and for the Two Treated Batches at 6 Months, Measured Using Image Analysis on Feulgen-Stained Gill Preparations $(n=\text { number of individuals sample })^{\text {a }}$

\begin{tabular}{|c|c|c|c|c|c|c|}
\hline Ploidy & $\begin{array}{l}\text { Batch } 1 \\
2 n \times 2 n \\
(n=10)\end{array}$ & $\begin{array}{l}\text { Batch } 2 \\
4 n \times 4 n \\
(n=10)\end{array}$ & $\begin{array}{l}\text { Batch } 3 \\
2 n \times 4 n+C B \\
(n=10)\end{array}$ & $\begin{array}{l}\text { Batch } 4 \\
4 n \times 2 n+C B \\
(n=20)\end{array}$ & $\begin{array}{l}\text { Batch } 3 \\
2 n \times 4 n+C B \\
(n=24)\end{array}$ & $\begin{array}{l}\text { Batch } 4 \\
4 n \times 2 n+C B \\
(n=24)\end{array}$ \\
\hline$\overline{A g e}$ & $4 \mathrm{mo}$ & $4 \mathrm{mo}$ & $4 \mathrm{mo}$ & $4 \mathrm{mo}$ & $6 \mathrm{mo}$ & $6 \mathrm{mo}$ \\
\hline $2 \mathrm{n}$ & $10(100 \%)$ & - & $3(30 \%)$ & - & $8(33 \%)$ & - \\
\hline $3 n$ & - & - & $3(30 \%)$ & $17(85 \%)$ & $11(46 \%)$ & $24(100 \%)$ \\
\hline $4 n$ & - & $10(100 \%)$ & $4(40 \%)$ & - & $5(21 \%)$ & - \\
\hline Other & - & - & - & $\begin{array}{l}\text { 2: between } 3 n-4 n \\
1: 3 n / 4 n \text { Mosaic }\end{array}$ & - & - \\
\hline
\end{tabular}

${ }^{a}$ Batches 1 and 2 represent the controls, which were diploid $(2 n)$ and tetraploid $(4 n)$, respectively. Batch 3 tests the new induction cross, which hypothetically produces tetraploids from $2 \mathrm{n}$ females $\times 4 \mathrm{n}$ males with cytochalasin $\mathrm{B}$ (CB) treatment. Batch 4 hypothetically produces pentaploids $(5 \mathrm{n})$ from $4 \mathrm{n}$ females $\times 2 \mathrm{n}$ males with CB treatment.

a: $2 n \times 4 n$ gives $3 n$
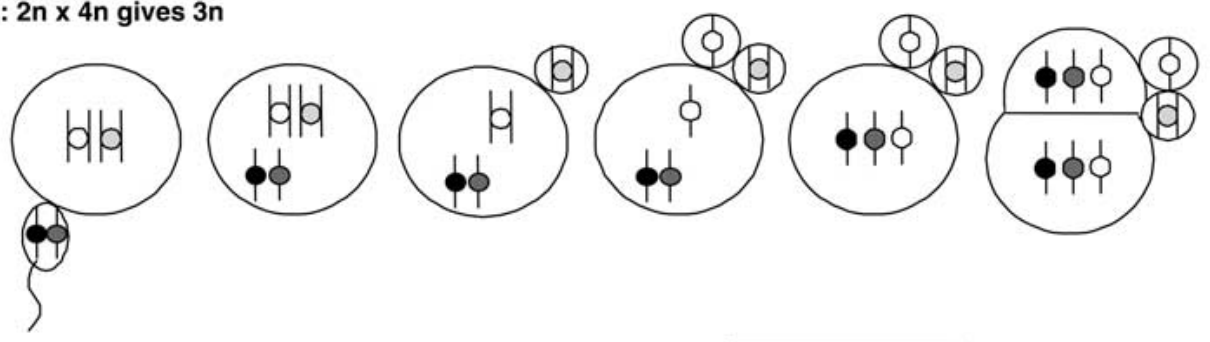

b: $2 n \times 4 n$ CB (PB2 inhibition gives $4 n$ )
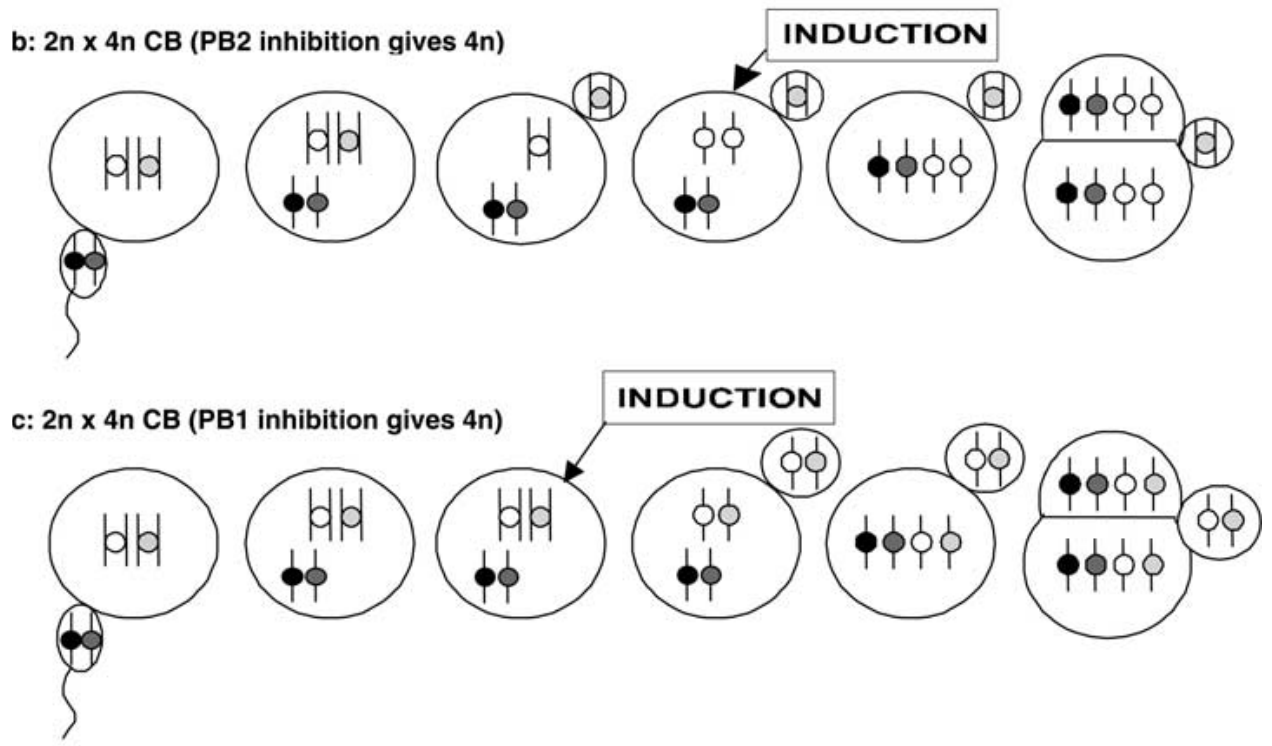

d: $2 n \times 4 n$ CB (PB1 and PB2 inhibition gives 6n) INDUCTION
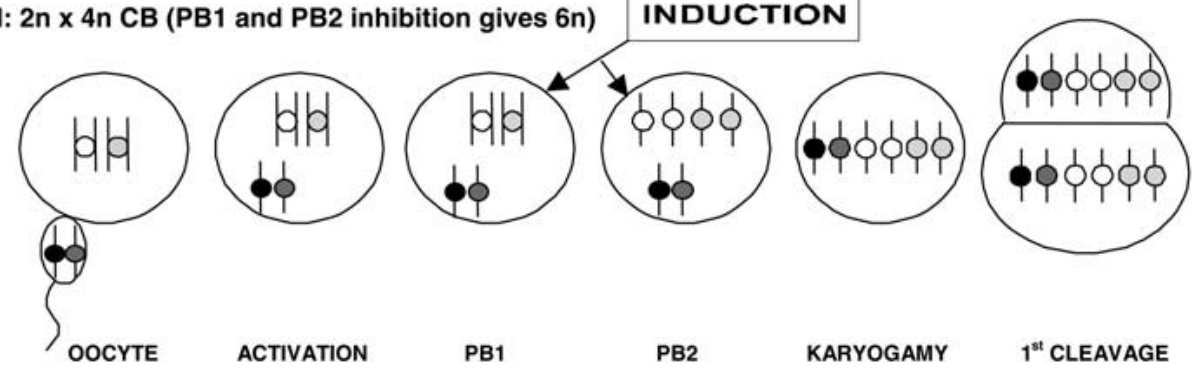

Fig. 5. Diagram of embryogenic events that could have led to the ploidy levels observed in the $2 n \times 4 n+C B$ cross. Triploids $(3 n)$ derived from diploid $(2 n)$ females $\times$ tetraploid $(4 n)$ males without polar body retention $(\mathbf{a})$; tetraploids (4n) from diploid $(2 n)$ females $\times$ tetraploid males with retention of the second polar body $(\mathbf{b})$. Retention of the first polar body on the same cross also gives tetraploids (c); retention of both polar bodies on the same cross would give hexaploids (6n) (d). 
The survival of $4 n$ oysters from the $2 n \times 4 n+C B$ cross to 6 months illustrates that large diploid eggs are capable of holding a tetraploid genome and successfully dividing and differentiating into a properly functioning animal. We therefore do not support the aspect of the cell-number deficiency hypothesis (Guo, 1991) which suggests that $4 \mathrm{n}$ cannot be produced from $2 \mathrm{n}$ eggs. However, we found larger diploid eggs and more variation in diploid egg size in general than in Guo's study, although the experimental circumstances in under which we derived our result differed from those of this author, as did the polyploidy induction methods used. With smaller eggs such as those used in this previous study (mean, $\sim 49.6 \mu \mathrm{m}$ ), or with higher ploidy levels relative to egg size, phenomena such as that described by this hypothesis could explain the lethality of high ploidy levels. An accurate comparison of the egg size measurements between these studies is difficult because our measurements were made before fertilization, rather than after as they were in Guo (1991). Differences that allowed our induction to succeed could be due to genetic differences in the stocks of Crassostrea gigas used. Larger egg size is the principal rationale that Guo and Allen (1994a) proposed for using triploid females for the generation of tetraploids, rather than the higher ploidy level of these eggs per se. Our study shows that selected diploid females can give suitable eggs for tetraploid induction. This suggests that other methods of manipulation, such as double PB retention, may be exploitable via selection of females with large eggs and therefore provide other means of inducing tetraploidy directly in C. gigas.

Problems of genome size relative to cell size may, however, be responsible for the disappearance of the multiple ploidy classes in the 24-hour sample of the $4 n \times 2 n+C B$ cross. Here also, differential development of embryos at the time of treatment means that individuals are at different stages, and there is some evidence that this phenomenon is greater in tetraploids (Guo and Allen 1997). Embryonic development was also slower overall in the $4 \mathrm{n} \times$ $2 n+C B$ cross (Figure 2, d) as in the $4 n \times 4 n$ (Figure 2, b), which would increase the likelihood of suppression of PB1 and double retention over PB2 alone (Figure 5, b-d). These scenarios would explain the variety of ploidies in the embryos studied at 24 hours (Figure $3, \mathrm{c}$ ). Although predominantly $5 \mathrm{n}$ at the larval stages we studied, the $4 n \times 2 n+C B$ batch produced virtually only $3 \mathrm{n}$ spat (Figures 3 , c, 4, e and 4, $\mathrm{f}$, Table 3). Production of $3 \mathrm{n}$ in our treated crosses may simply have arisen from animals on which the chemical treatment had no effect. However, this does not explain the gynogenetic individuals in the
$4 n \times 2 n+C B$ (discussed below). Production of $3 n$ batches by $2 n \times 4 n$ and $4 n \times 2 n$ untreated crosses would have provided another level of comparison for the treated lots. We did not integrate these crosses into the present experimental design because of limits on the quantity of female gametes, hatchery tank space, and time for the tests subsequently made. A future study could target comparison of one interploidy cross type (e.g., $2 \mathrm{n} \times 4 \mathrm{n}$ ) with and without $\mathrm{CB}$ treatment.

A final alternative hypothesis of variable ploidy level in offspring is heterogeneity of ploidy of gametes. In another study we tested the ploidy of spermatozoa from tetraploids using image analysis, and these were confirmed to be diploid (McCombie et al., in press). We therefore did not expect a problem in this area. The $4 n \times 4 n$ control produced all tetraploids according to our tests, which also indicates that the parental gametes were normal and homogeneous. However, a finer scale study is required to better examine both male and female gametes by a method such as flow cytometry, so as to increase accuracy for the lower ploidy level of gametes. Were tetraploid males to produce a percentage of haploid (rather than expected diploid) spermatozoa, this could give lower offspring ploidy levels than expected. This subject is also important because spermatozoa from tetraploids form the basis of triploid breeding in hatcheries at present, and their quality is therefore important for the aquaculture industry. Because hypotetraploid cells $1<40$ chromosomes per cell) have been observed in tissues of some tetraploids (McCombie et al., in press), it is possible that this can also occur in gametes and therefore represents an important subject for further study.

In this study no clear evidence of aneuploids (cells containing irregular chromosome numbers rather than multiples of the haploid number) was revealed. Chromosome preparations would be necessary to investigate this point in detail because image analysis, although it can detect some mosaic chromosome conditions (personal observations), does not allow this degree of accuracy. In triploid induction, the higher number of anomalies observed from blocking PB1, rather than PB2, is thought to be due to the subsequent passage through meiosis II that the first group must experience (Gérard et al., 1999), which may cause unbalanced chromosome segregation at this point (Guo et al., 1992b; Yang et al., 2000). For this reason, more abnormal animals might be expected in the induction of tetraploids from triploids by PB1 suppression (Guo and Allen, 1994a). Some of these intermediate $2 n-4 n$ aneuploids, produced by PB1 suppression, died in large 
numbers, while others showed normal growth. A chromosome study in the present circumstances would allow us to detect any surviving aneuploids produced in our study.

Changes in Ploidy Level Over Time. In both treated crosses, ploidy of the treated batches changed over time. The $2 n \times 4 n+C B$ started predominantly $4 \mathrm{n}$ at 24 hours but showed a large $3 \mathrm{n}$ peak by 72 hours. The fixed juveniles tested from this cross were a mixture of ploidies. The $4 n \times 2 n+C B$ results started extremely mixed-2n-9n at 24 hours-but quickly stabilized with the majority at $5 \mathrm{n}$. Juveniles, however, were only $3 \mathrm{n}$. A decrease in pentaploids replaced by triploids over the larval period was also observed by Scarpa et al. (1993) and could be due to differential survival. Change in the ploidy of individual animals has also been suggested (Peruzzi and Guo, 2002). In the present study, the dramatic drop in larval numbers that accompanied the change in ploidy between 24 and 72 hours suggests that it may well be differential mortality of ploidy classes that accounts for the changes.

Less change took place between the 2 measurements made on juveniles at 4 and 6 months; the main classes present remained the same in the different batches. These sampling dates are quite close together, and later samples would be necessary to detect a real evolution. Ploidy composition can change over time owing differential mortality or the phenomenon of reversion (chromosome loss). There may also be differences related to size (Scarpa et al., 1993), so that the deliberate selection of large animals to facilitate tests could favor more vigorously growing ploidy classes such as triploids and thus distort the evaluation of a group. The animals in the present study have now been separated into size classes to avoid the increasing influence of such differences due to competition. The differences of ploidy with animal size would make an interesting subject for further study.

Between the larval and postfixation samplings, however, classes appeared that did not represent a high percentage in the early samplings at 24 and 72 hours, notably the occurrence of $2 n$ in $2 n \times 4 n+C B$ and $3 n$ in $4 n \times 2 n+C B$. Metamorphosis and fixation are crucial stages in the development of young oysters and may represent a barrier to further development of certain ploidy classes such as $5 \mathrm{n}$. Other classes may subsequently represent a greater proportion of the batches.

The Generation of Gynogenetic Triploids. The microsatellite-based parentage analyses revealed that a small proportion of the triploids generated in the $4 n \times 2 n$ CB cross were gynogenetic. The eggs in this cross came from tetraploids and were therefore also $4 \mathrm{n}$ at the start of the experiment (before reduction division). The simplest explanation for the gynogens is an unequal division at meiosis I induced by the $\mathrm{CB}$, followed by the expulsion of an irregular polar body containing 10 chromosomes. Irregular divisions at meiosis I have already been observed in C. gigas (Guo et al., 1992a) and are one of the major drawbacks of using PB1 inhibition for the generation of triploids from diploid parents. Either the nonpenetration of sperm or its subsequent rejection is also necessary for gynogenesis. Nonpenetration may be a side effect of CB (Longo, 1978; Ledu and McCombie, 2003). We have also observed unusual patterns of $\mathrm{PB}$ expulsion in other ploidy manipulation experiments; on rare occasions these included the expulsion of most or all the genetic material in one PB (Ledu and McCombie, 2003). The effects of CB may therefore be diverse. Future study could usefully compare triploids from both treated crosses with triploids from untreated crosses of the same parents.

Androgenesis, which was detected in one animal in the $2 n \times 4 n+C B$ treatment, has already been observed in this species in crosses with diploid sperm (Guo and Allen, 1997). The phenomenon occurred in an untreated diploid-tetraploid cross and was thus spontaneous. This result may therefore be independent of the CB treatment, although further study would be required to ascertain what influence the chemical may have had.

Further application of molecular biology tools could help identify any selective rejection of maternal or paternal genomes during PB expulsion.

Prospects for Uses of the Animals Generated and Methodology Used. The method we describe allows genetic material from diploids to be introgressed into tetraploid lines without passing by a triploidization step or requiring the selection of fertile female triploids. This means that desirable traits could be selected in diploids (outside of the quarantine conditions required for keeping tetraploids) and then introduced into tetraploids. Because this method $(2 n \times 4 n+C B)$ produces a mixture of ploidies, a sorting step is necessary by nondestructive biopsy of gill tissue under $\mathrm{MgCl}$ anesthesia. Such sorting does not represent an additional step as it is necessary for all $4 \mathrm{n}$ used in $4 \mathrm{n} \times 4 \mathrm{n}$ crosses, or as progenitors for $3 \mathrm{n}$ production, to eliminate any non$4 \mathrm{n}$ animals that may be in a batch /due to reversion, for example; McCombie et al., in press) .

The unusual observation of gynogenetic triploids from the $4 n \times 2 n+C B$ may point the way to a means 
of production of animals of a uniform genetic background for genetic studies. Gynogenetic diploids have already been produced (Guo et al., 1993), but they showed limited viability, and the authors suggest this was due to deleterious recessive alleles in the homozygous state. Triploid gynogens may avoid this problem but would in consequence be less genetically uniform.

Future work will focus on more precise timing of CB treatments of $2 n \times 4 n+C B$ crosses to increase the yield of tetraploids. Shortened exposure times on eggs of single females may produce better results, as would monitoring of a subsample of embryos for polar body expulsion (Eudeline et al., 2000). Chromosome preparations would help reveal the nature of any aneuploids and mosaics produced, and further tests of ploidy levels through time as the offspring age would help evaluate the long-term stability of tetraploid progenies produced in this way.

\section{Acknowledgments}

We thank the technical staff at Ifremer La Tremblade for the care and preparation of the parental animals used in this study and Florence Cornette for practical assistance during the larval rearing period. Some preliminary experiments leading to this study were conducted and tested by Raphaël Brizard. Delphine Lallias and Nicolas Taris gave invaluable advice on the application of microsatellites.

\section{References}

1. Boudry P, Chatain B (1999) Triploidy in mariculture: status and perspectives Position paper adopted by the Working Group on the Application of Genetics in Fisheries and Mariculture (WGAGFM), Reykjavik, Iceland April 12-15 ICES CM 1999/F1, pp 7-20

2. Chourrout D, Chevassus B, Kreig R, Happe A, Burger G, Renard P (1986) Production of second generation triploid and tetraploid rainbow trout by mating tetraploid males and diploid females: potential of tetraploid fish. Theor Appl Gen 72, 193-286

3. Downing SL, Allen SK Jr (1987) Induced triploidy in the Pacific oyster Crassostrea gigas: optimal treatments with cytochalasin B depend on temperature. Aquaculture 61, 1-15

4. Dube F, Schmidt T, Johnson CH, Epel D (1985) The hierachy of requirements for an elevated intracellular $\mathrm{pH}$ during early development of sea urchin embryos. Cell 40, 657-666

5. Ernande B, Clobert J, McCombie H, Boudry P (2003) Genetic polymorphism and trade-offs in the early lifehistory strategy of the Pacific oyster, Crassostrea gigas (Thunberg, 1795): a quantitative genetics study. J Evol Biol 16, 399-141
6. Ernande B, Boudry P, Clobert I, Haure J (2004) Plasticity in resource allocation based life history traits of the Pacific oyster, Crassostrea gigas, I: spatial variation in food abundance. J Evol Biol 17, 342-356

7. Eudeline B, Allen SK Jr, Guo X (2000) Optimization of tetraploid induction in Pacific oysters, Crassostrea gigas, using first polar body as a natural indicator. Aquaculture 187, 73-84

8. Gérard A, Naciri Y, Peignon J-M, Ledu C, Phelipot Noiret PC, Peudenier I, Grizel H (1994) Image analysis: a new method for estimating triploidy in commercial bivalves. Aquacult Fish Manage 25, 697-708

9. Gérard A, Ledu C, Phelipot R, Naciri-Graven Y (1999) The induction of MI and MII triploids in the Pacific oyster Crassostrea gigas with 6-DMAP or CB. Aquaculture 174, 229-242

10. Guo X (1991) Studies on tetraploidy induction in the Pacific oyster, Crassostrea gigas. Seattle, PhD dissertation University of Washington

11. Guo X, Allen SK Jr (1994a) Viable tetraploids in the Pacific oyster (Crassostrea gigas Thunberg) produced by inhibiting polar body 1 in eggs from triploids. Mol Mar Biol Biotec nol 3, 42-50

12. Guo X, Allen SK Jr (1994b) The reproductive potential and genetics of triploid Pacific oyster Crassostrea gigas (Thunberg). Biol Bull 187, 309-318

13. Guo X, Allen SK Jr (1997) Sex and meiosis in autotetraploid Pacific oyster, Crassostrea gigas. Genome 40, 397-405

14. Guo X, Hershberger WK, Cooper K, Chew K (1992a) Genetic consequences of blocking polar body I with cytochalasin B in fertilized eggs of the Pacific oyster, Crassostrea gigas, I: ploidy of resultant embryos. Biol Bull 183, 381-386

15. Guo X, Hershberger WK, Cooper K, Chew K (1992b) Genetic consequences of blocking polar Body I with cytochalasin B in fertilized eggs of the Pacific oyster, Crassostrea gigas, II: segregation of chromosomes. Biol Bull 183, 387-393

16. Guo X, Hershberger WK, Cooper K, Chew K (1993) Artificial gynogenesis with ultraviolet light-irradiated sperm in the Pacific oyster, Crassostrea gigas, I: induction and survival. Aquaculture 113, 201-214

17. Guo X, Debrosse GA, Allen SK Jr (1996) All triploid oysters (Crassostrea gigas Thunberg) produced by mating tetraploids and diploids. Aquaculture 142, 149-161

18. Huvet A, Boudry P, Ohresser M, Delsert C, Bonhomme F (2000) Variable microsatellites in the Pacific cupped oyster Crassostrea gigas and other cupped oyster species. Anim Genet 37, 71-72

19. Kearsey MJ, Pooni HS (1996) The Genetical Analysis of Quantitative Traits. (Cheltenham, UK: Stanley Thornes (Publishers) Ltds)

20. Langdon C, Evans F, Jacobson D, Blouin M (2003) Yields of cultured Pacific oysters, Crassostrea gigas Thunberg improved after one generation of selection. Aquaculture 220, 227-244

21. Ledu C, McCombie H (2003) Effects of cytochalasin B on fertilization and ploidy in the Pacific oyster Crassostrea gigas. Invertebr Reprod Dev 42, 131-137 
22. Longo FJ (1978) Effects of cytochalasin B on sperm-egg interactions. Dev Biol 67, 157-173

23. Longwell AC, Stiles SS (1968) Fertilization and completion of meiosis in spawned eggs of the American oyster Crassostrea virginica. Caryologia 21, 65-73

24. Magoulas A, Gjetvaj B, Terzoglou V, Zouros E (1998) Polymorphic microsatellites in the Japanese oyster, Crassostrea gigas (Thunberg). Anim Genet 29, 63-72

25. McCombie H, Lapegue S, Cornette F, Ledu C, Boudry, $\mathrm{P}(\mathrm{xxxx})$ Chromosome loss in bi-parental progenies of tetraploid Pacific oyster Crassostrea gigas. Aquaculture (in press)

26. Nell JA (2002) Farming triploid oysters. Aquaculture 210, 69-88

27. Nell JA, Smith IR, McPhee CC (2000) The Sydney rock oyster Saccostrea glomerata (Gould 1850) breeding programme: progress and goals. Aquacult Res 31, 4549

28. Pandian TJ, Koteeswaran R (1998) Ploidy induction and sex control in fish. Hydrobiologia 384, 167-243

29. Peruzzi S, Guo X (2002) Tetraploid induction by meiosis inhibition with cytochalasin B in the dwarf surfclam, Mulima lateris Say: effects of temperature. J Shellfish Res 21, 677-684

30. Que H, Guo X, Zhang F, Allen SK Jr (1997) Chromosome segregation in fertilized eggs from triploid oyster,
Crassostrea gigas (Thunberg), following inhibition of polar body 1. Biol Bull 193, 14-19

31. Robert R, Gérard A (1999) Bivalve hatchery technology: the current situation for the Pacific oyster Crassostrea gigas and the scallop Pecten maximus in France. Aquat Living Resour 12, 121-130

32. Scarpa J, Wada KT, Komura A (1993) Induction of tetraploidy in mussels by suppression of polar body formation. Nippon Suisan Gakkaishi 59, 2017-2023

33. Sheridan AK (1997) Genetic improvement of oyster production-a critique. Aquaculture 153, 165-179

34. Stanley JG, Hidu H, Allen SK Jr (1981) Polyploidy induced in the American oyster, Crassostrea virginica, with cytochalasin B. Aquaculture 37, 147-155

35. Ward RD, English LJ, Mcgoldrick DJ, Maguire GB, Nell JA, Thompson PA (2000) Genetic improvement of the Pacific oyster (Crassostrea gigas Thunberg) in Australia. Aquacult Res 31, 35-44

36. Yamamoto S, Sugawara Y, Nomura T, Oshino A (1988) Induced triploidy in Pacific oyster Crassostrea gigas, and performance of triploid larvae. Tohoku J Agric Res 39, 47-59

37. Yang H, Que H, He Y, Zhang F (2000) Chromosome segregation in fertilized eggs from zhikong scallop Chlamys farreri (Jones \& Preston) following polar body 1 inhibition with cytochalasin B. J Shellfish Res 19, 101-105 